\title{
Soft-Decision Demodulation Design for COVQ over White, Colored, and ISI Gaussian Channels
}

\author{
Nam Phamdo, Senior Member, IEEE, and Fady Alajaji, Senior Member, IEEE
}

\begin{abstract}
In this work, the design of a $q$-bit (scalar and vector) soft-decision demodulator for Gaussian channels with binary phase-shift keying modulation is investigated. The demodulator is used in conjunction with a soft-decision channel-optimized vector quantization (COVQ) system. The COVQ is constructed for an expanded $(q>1)$ discrete channel consisting of the concatenation of the modulator, the Gaussian channel, and the demodulator. It is found that as the demodulator resolution $q$ increases, the capacity of the expanded channel increases, resulting in an improvement of the COVQ performance. Consequently, the soft-decision demodulator is designed to maximize the capacity of the expanded channel. Three Gaussian channel models are considered as follows: 1) additive white Gaussian noise channels; 2) additive colored Gaussian noise channels; and 3) Gaussian channels with intersymbol interference. Comparisons are made with a) hard-decision COVQ systems, b) COVQ systems which utilize interleaving, and c) an unquantized $(q=\infty)$ soft-decision decoder proposed by Skoglund and Hedelin. It is shown that substantial improvements can be achieved over COVQ systems which utilize hard-decision demodulation and/or channel interleaving. The performance of the proposed COVQ system is comparable with the system by Skoglund and Hedelin-though its computational complexity is substantially less.
\end{abstract}

Index Terms-Additive white/colored Gaussian noise, capacity of discrete channels, combined source-channel coding, COVQ, ISI channels, soft-decision decoding.

\section{INTRODUCTION}

$\mathbf{S}$ INCE the groundbreaking paper of Shannon [17], the source and channel coding components of a communication system have been designed and implemented separately (in tandem). This separation of source and channel coding results in no loss of optimality provided infinite coding delay and unlimited system complexity are allowed [17]. However, in practical communication systems, where the amount of tolerated delay and complexity is constrained, numerous works have shown that combined source-channel coding systems can

Paper approved by E. Ayanoglu, the Editor for Communication Theory and Coding Application of the IEEE Communications Society. Manuscript received March 12, 1999; revised February 8, 2000. The work of N. Phamdo was supported in part by Northrop Grumman Corporation. The work of F. Alajaji was supported in part by the Natural Sciences and Engineering Research Council of Canada (NSERC) under Grant OGP0183645. This paper was presented in part at the Conference on Information Sciences and Systems, Princeton, NJ, March 1996, and at the IEEE International Symposium on Information Theory, Ulm, Germany, June 1997.

N. Phamdo is with the Department of Electrical and Computer Engineering, State University of New York, Stony Brook, NY 11794-2350 USA (e-mail: phamdo@ece.sunysb.edu).

F. Alajaji is with the Department of Mathematics and Statistics and the Department of Electrical and Computer Engineering, Queen's University, Kingston, ON K7L 3N6, Canada (e-mail: fady@mast.queensu.ca).

Publisher Item Identifier S 0090-6778(00)07521-8. significantly outperform traditional tandem coding systems, in particular with regards to the design of vector quantization schemes for noisy communication channels (e.g., [1], [2], [4], [5], [7]-[10], [12]-[14], [16], [18], [19], [21], [22]).

The majority of the previous work on the study of vector quantization for noisy channels consider discrete channel models (with the exception of [1], [9], [12], [16], [18], [19], [21], and [22]); i.e., channels used in conjunction with hard-decision demodulation. Furthermore, with the exception of [9], [14], and [19], these works focus on memoryless channel models.

In this paper, we incorporate the use of the soft-decision channel information in the design of combined source-channel coding systems for noisy channels. More specifically, we introduce a channel-optimized vector quantization (COVQ) scheme [10], [4] for additive Gaussian noise channels with binary phase-shift keying (BPSK) and soft-decision demodulation. This is achieved by (scalar or vector) quantizing the channel output via a $q$-bit soft-decision demodulator (where $q>1$ ), and designing a COVQ system for the resulting expanded discrete channel which consists of the concatenation of the modulator, the Gaussian channel, and the demodulator. We consider Gaussian noise channel models both with and without memory as follows: 1) additive white Gaussian noise (AWGN) channels; 2) additive colored Gaussian Noise (ACGN) channels; and 3) Gaussian channels with intersymbol interference (ISI). In the case of the Gaussian channels with memory, unlike traditional systems that employ standard channel interleaving or linear/nonlinear equalization techniques, we utilize the statistical correlation of the channel in the design of our soft-decision COVQ system. In other words, we design a COVQ scheme that exploits both the (intrablock) channel memory as well as the channel soft-decision information. Numerical results demonstrate that substantial improvements can be achieved over COVQ schemes designed for hard-decision channels and fully interleaved channels.

In previous related work, the authors presented a similar soft-decision COVQ system for memoryless Rayleigh fading channels in [1]. Optimal minimum mean-squared error (MMSE) sequential soft decoders for vector quantizers were investigated for memoryless Rayleigh fading channels in [12] and for Gaussian channels with linear memory in [9]. In [18] and [19], optimal and suboptimal Hadamard-based soft decoders were proposed and implemented for AWGN, Rayleigh, and ISI channels. While our soft-decision COVQ scheme has a higher storage requirement than the Hadamard-based soft decoder of [18] and [19], its computational complexity is considerably lower. 


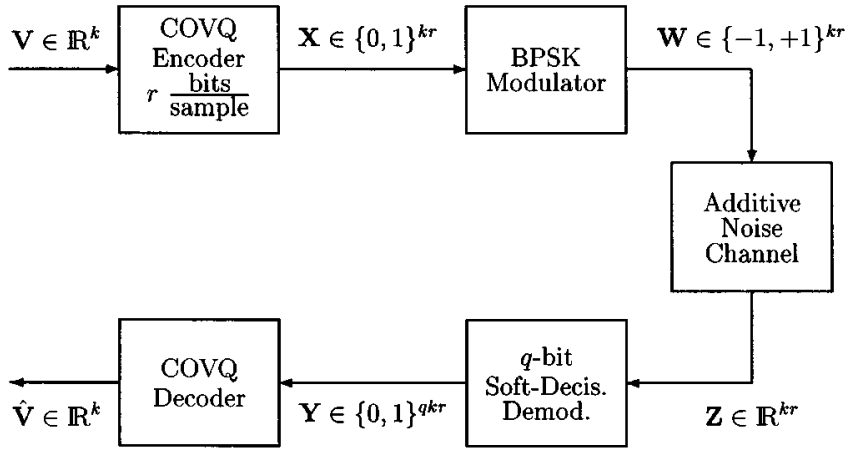

Fig. 1. Block diagram of the COVQ system.

The rest of this paper is organized as follows. In Section II, a soft-decision COVQ system for BPSK-modulated AWGN channels is proposed, analyzed, and implemented. Its performance is also compared to that of the Hadamard-based decoder in [18]. In Section III, a soft-decision COVQ scheme for ACGN without noise whitening is studied. Scalar and vector soft-decision quantization systems for ACGN channels with noise whitening are examined in Section IV. In Section V, quantization over ISI channels is addressed and performance comparisons to the scheme in [19] are presented. Finally, conclusions are stated in Section VI.

\section{AWGN CHANNELS}

\section{A. DMC Channel Model}

Consider the following combined source-channel coding system (cf. Fig. 1). The input source $\mathbf{V}$ is a $k$-dimensional real vector, and the COVQ operates at a rate of $r$ bits per source dimension. For each input vector, the encoder produces a binary vector $\mathbf{X} \in\{0,1\}^{k r}$ for transmission, where $k r$ is assumed to be an integer. Each of the $k r$ bits of $\mathbf{X}$ is BPSK modulated, and the output $\mathbf{W} \in\{-1,+1\}^{k r}$ is transmitted over an AWGN channel according to

$$
Z_{n}=W_{n}+U_{n}
$$

$n=1,2, \cdots, k r$, where $W_{n} \in\{-1,+1\}$ is the BPSK signal of unit energy, and $U_{n}$ is a zero-mean independent and identically distributed (i.i.d.) Gaussian random process with variance $N_{0} / 2$.

At the receiver, each component of the received vector $\mathbf{Z}$ is demodulated via a $q$-bit uniform scalar quantizer with quantization step $\Delta$ to yield $\mathbf{Y} \in\{0,1\}^{q k r}$. Thus, for each $k$-dimensional source vector, $q k r$ bits are produced at the demodulator output. These bits are then passed to the COVQ decoder to determine the estimate $\hat{\mathbf{V}} \in \mathbb{R}^{k}$.

\section{B. Capacity and Soft-Decision Quantizer Design}

We observe that the concatenation of the modulator, channel, and demodulator constitutes indeed a $2^{k r}$-input, $2^{q k r}$-output discrete memoryless channel (DMC). Since the noise is i.i.d., this channel is equivalent to a binary-input, $2^{q}$-output DMC used $k r$ times. Its channel transition probability matrix can hence be computed in terms of the quantization step $\Delta$, the channel signal-to-noise ratio (SNR), and the complementary error function. More specifically, if $\mathcal{X}=\{0,1\}$ and $\mathcal{Y}=\left\{0,1,2, \cdots, 2^{q}-1\right\}$, then the transition probability matrix $\Pi$ is given by

$$
\Pi=\left[\pi_{i j}\right], \quad i \in \mathcal{X}, \quad j \in \mathcal{Y}
$$

where

$$
\begin{aligned}
\pi_{i j} & \triangleq P(Y=j \mid X=i) \\
& =Q\left(\left(T_{j-1}-(2 i-1)\right) \sqrt{\mathrm{SNR}}\right) \\
& -Q\left(\left(T_{j}-(2 i-1)\right) \sqrt{\mathrm{SNR}}\right) .
\end{aligned}
$$

Here, SNR $=E\left[W_{n}^{2}\right] / E\left[U_{n}^{2}\right]=\left(2 / N_{0}\right)$

$$
Q(x)=\frac{1}{\sqrt{2 \pi}} \int_{x}^{\infty} \exp \left\{-t^{2} / 2\right\} d t
$$

is the complementary error function, and $\left\{T_{j}\right\}$ are the thresholds of the receiver's soft-decision quantizer $\alpha(\cdot)$, which is defined as

$$
\alpha(z)=j, \quad \text { if } z \in\left(T_{j-1}, T_{j}\right)
$$

$j=0,1, \cdots, 2^{q}-1$. In this work, we assume that the thresholds $\left\{T_{j}\right\}$ are uniformly spaced with step-size $\Delta$ such that

$$
T_{j}= \begin{cases}-\infty, & \text { if } j=-1 \\ \left(j+1-2^{q-1}\right) \Delta, & \text { if } j=0,1, \cdots, 2^{q}-2 \\ +\infty, & \text { if } j=2^{q}-1 .\end{cases}
$$

We observe that the above two-input, $2^{q}$-output DMC is "weakly" symmetric in the sense that its transition probability matrix $\Pi$ can be partitioned (along its columns) into symmetric arrays - where a symmetric array is defined as an array having the property that all its rows are permutations of each other, and all its columns are permutations of each other [6], [3]. The symmetry property implies the fact that the capacity of this channel is achieved by a uniform input distribution [6]. Its capacity can therefore be easily computed by evaluating the mutual information between $X$ and $Y, I(X ; Y)$, using a uniform distribution on $X$. In Table $\mathrm{I}(\mathrm{a})$, we display the channel capacity for different values of $q$ and the channel SNR. For each channel SNR, we numerically select the value of the quantization step $\Delta$ which yields the maximum capacity of the binary-input $2^{q}$-output DMC. The motivation for this approach is twofold: i) an increase in channel capacity typically would result in an increase in overall system performance [measured in term of source signal-to-distortion ratio (SDR)] and ii) optimizing the soft-decision quantizer under the maximum capacity criterion is much simpler than under the maximum SDR criterion. Note that the capacity increases with $q$ (as 
TABLE I

(a) CAPACity $C$ (IN Bits/Channel Use) OF Two-InPut, $2^{q}$-OutPut DMC DERIVED FROM BPSK-MODULATED AWGN CHANNEL WITH $q$-BIT SOFT-DECISION DEMODULATION; $\Delta=\Delta$ (SNR) IS OPTIMAL Quantization SteP. (b) Source SDR (IN Decibels) Performances OF COVQ SYSTEM IN AWGN CHANNEL FOR DIFFERENT VALUES OF $q$ (Number OF SOFT-DeCISION BITS); MEMORYLESS GaUsSiAn SOURCE; $r=2$ BITS/SAMPLE; DIMENSION $k=2$. NUMBERS IN BRACKETS InDiCATE THE OPTIMAL PERFoRMANCE THEORETICALLY ATTAINABLE (OPTA) FOR THE MEMORYLESS GAUSSIAN SOURCE AND DMC

\begin{tabular}{c|c|c|c|c|c|c|c}
\hline Channel & $q=1$ & \multicolumn{2}{|c|}{$q=2$} & \multicolumn{2}{c|}{$q=3$} & \multicolumn{2}{c}{$q=4$} \\
\cline { 2 - 8 } SNR & $C$ & $C$ & $\Delta$ & $C$ & $\Delta$ & $C$ & $\Delta$ \\
\hline \hline$\infty$ & 1.000 & 1.000 & 1.000 & 1.000 & 1.000 & 1.000 & 1.000 \\
8.0 & 0.947 & 0.971 & 0.27 & 0.975 & 0.15 & 0.976 & 0.08 \\
6.0 & 0.842 & 0.898 & 0.37 & 0.908 & 0.20 & 0.911 & 0.11 \\
4.0 & 0.687 & 0.770 & 0.49 & 0.788 & 0.27 & 0.793 & 0.15 \\
3.0 & 0.602 & 0.693 & 0.57 & 0.713 & 0.31 & 0.718 & 0.18 \\
2.0 & 0.518 & 0.612 & 0.65 & 0.634 & 0.36 & 0.640 & 0.20 \\
1.0 & 0.440 & 0.532 & 0.75 & 0.554 & 0.42 & 0.560 & 0.23 \\
0.0 & 0.369 & 0.455 & 0.86 & 0.477 & 0.48 & 0.483 & 0.27 \\
-1.0 & 0.306 & 0.385 & 0.98 & 0.406 & 0.55 & 0.412 & 0.31 \\
-2.0 & 0.252 & 0.322 & 1.12 & 0.341 & 0.63 & 0.347 & 0.36 \\
-3.0 & 0.206 & 0.267 & 1.28 & 0.284 & 0.72 & 0.289 & 0.41 \\
\hline \multicolumn{6}{|c|}{ (a) }
\end{tabular}

\begin{tabular}{c|c|c|c|c}
\hline \hline $\begin{array}{c}\text { Channel } \\
\text { SNR }\end{array}$ & $q=1$ & $q=2$ & $q=3$ & $q=4$ \\
\hline$\infty$ & $9.57[12.04]$ & $9.57[12.04]$ & $9.57[12.04]$ & $9.57[12.04]$ \\
8 & $8.64[11.40]$ & $8.76[11.69]$ & $8.82[11.74]$ & $8.84[11.75]$ \\
6 & $6.89[10.14]$ & $7.21[10.81]$ & $7.32[10.93]$ & $7.35[10.97]$ \\
4 & $5.17[8.27]$ & $5.74[9.27]$ & $5.87[9.49]$ & $5.91[9.55]$ \\
3 & $4.38[7.25]$ & $5.06[8.34]$ & $5.21[9.29]$ & $5.25[8.64]$ \\
2 & $3.77[6.24]$ & $4.36[7.37]$ & $4.50[7.63]$ & $4.54[7.71]$ \\
1 & $3.17[5.28]$ & $3.71[6.41]$ & $3.85[6.67]$ & $3.89[6.74]$ \\
0 & $2.66[4.44]$ & $3.14[5.48]$ & $3.27[5.74]$ & $3.30[5.82]$ \\
-1 & $2.21[3.68]$ & $2.69[4.64]$ & $2.81[4.89]$ & $2.84[4.96]$ \\
-2 & $1.82[3.03]$ & $2.26[3.88]$ & $2.37[4.11]$ & $2.40[4.18]$ \\
-3 & $1.50[2.48]$ & $1.88[3.21]$ & $1.98[3.42]$ & $2.01[3.48]$ \\
\hline \multicolumn{4}{|c}{}
\end{tabular}

(b)

expected) ${ }^{1}$ however, most of the capacity increase over the hard-decision demodulation system $(q=1)$ is achieved for $q=2$. Furthermore, the soft-decision information significantly increases the channel capacity during severe channel conditions; for example, at a channel SNR of $-3 \mathrm{~dB}$, the capacity increases by $40 \%$ (from $q=1$ to $q=4$ ).

\section{Numerical Results and Discussion}

As in [1], we employ the transition matrix $\Pi$ of the above $\left(2^{k r}\right.$-input, $2^{q k r}$-output) DMC to design a COVQ. The COVQ is obtained via the algorithm described in [1] and [4]. It consists of an iterative algorithm that results in a locally optimal solution. As discussed in [1], our scheme has no decoding computational requirements (as opposed to [19]); although the codebook size is larger than the codebook in [19].

In Table I(b), we present numerical results for the scheme in Fig. 1 when the source is memoryless Gaussian. The results are given in terms of the source SDR. The numbers in brackets indicate the optimal performances theoretically attainable (OPTA) obtained by evaluating $D(r C)$, where $D(\cdot)$ is the distortion-rate function of the source (for the squared-error distortion measure),

\footnotetext{
${ }^{1}$ Indeed, as $q \rightarrow \infty$, the capacity of the DMC monotonically converges to the capacity of the binary input AWGN channel with unquantized output [20].
}

TABLE II

SOURCE SDR (IN DeCibels) Performances of COVQ SySTEM IN AWGN

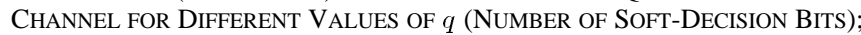
Memoryless Gaussian Source; $r=2$ Bits/Sample. Size OF COVQ DECODER TABLE IS FiXed AT 256. Numbers IN BRACKETS INDICATE THE OPTIMAL PERFormance THEORETICALLY ATTAINABLE (OPTA) FOR THE MEMORYLESS GAUSSIAN SOURCE AND DMC

\begin{tabular}{c|c|c|c}
\hline \hline $\begin{array}{c}\text { Channel } \\
\text { SNR }\end{array}$ & $k=4, q=1$ & $k=2, q=2$ & $k=1, q=4$ \\
\hline$\infty$ & $10.19[12.04]$ & $9.57[12.04]$ & $9.31[12.04]$ \\
8 & $8.90[11.40]$ & $8.76[11.69]$ & $8.59[11.75]$ \\
6 & $7.31[10.14]$ & $7.21[10.81]$ & $7.08[10.97]$ \\
4 & $5.50[8.27]$ & $5.74[9.27]$ & $5.19[9.55]$ \\
3 & $4.67[7.25]$ & $5.06[8.34]$ & $4.32[8.64]$ \\
2 & $3.93[6.24]$ & $4.36[7.37]$ & $3.57[7.71]$ \\
1 & $3.28[5.28]$ & $3.71[6.41]$ & $3.24[6.74]$ \\
0 & $2.73[4.44]$ & $3.14[5.48]$ & $3.17[5.82]$ \\
-1 & $2.26[3.68]$ & $2.69[4.64]$ & $2.71[4.96]$ \\
-2 & $1.86[3.03]$ & $2.26[3.88]$ & $2.29[4.18]$ \\
-3 & $1.52[2.48]$ & $1.88[3.21]$ & $1.92[3.48]$ \\
\hline
\end{tabular}

and $C$ is the capacity of the DMC derived from the BPSK-modulated AWGN channel. The rate is $r=2$ bits/sample and the dimension is $k=2$. Throughout the paper, we assume that the exact channel parameters are known to the encoder and decoder. We used 80000 training vectors in the COVQ design program. The numerical results are obtained using the vectors inside this training set. Note that the results for $q=1$ correspond to hard-decision demodulation. In this case, the DMC is derived from $k r$ uses of a binary symmetric channel (BSC) with crossover probability $Q(\sqrt{\mathrm{SNR}})$. Thus, for $q=1$, the results are nearly identical to those reported in [4] for the BSC. Observe from Table I(b) that the system performance increases as $q$ increases (and that most of the gain is achieved at $q=2$ ). In this case, the largest improvement is $0.87-\mathrm{dB}$ SDR occurring at $3-\mathrm{dB}$ channel SNR. Also, it can be remarked that at low channel SNR, the $q=4$ bit soft-decision scheme is approximately $1.3 \mathrm{~dB}$ in channel SNR better than the hard-decision scheme $(q=1)$; this is in contrast to the typical 2-dB coding gain obtained over AWGN channels at high channel SNR in soft-decision coded modulation systems.

The cost of doing soft-decision demodulation is increased complexity. The main complexity is due to the amount of memory needed to store the look-up table in the COVQ decoder. This table includes $2^{q k r}$ vectors-each with dimension $k$. The size of the table increases exponentially with $q$. It is hence interesting to study the behavior of the proposed system when the size of this table is constrained. In Table II, we provide numerical results for the COVQ system when $r=2$ and $q k=4$. In this case, the table in the COVQ decoder will always consist of 256 vectors (though the dimension of each vector is $k$ which varies). It can be seen that only at very high channel SNRs $(\geq 6 \mathrm{~dB})$, the hard-decision scheme slightly outperforms the soft-decision schemes. However, at lower SNRs, the soft-decision schemes are superior. Furthermore, the soft-decision schemes have lower computational and storage complexity in the encoder. Also, the dimension of the vectors in the decoder table is smaller.

In Table III(a) and (b), numerical results are provided for the case where the source is Gauss-Markov with correlation 
TABLE III

SOURCE SDR (IN DECIBELS) PERFORMANCES OF COVQ SYSTEM IN AWGN CHANNEL FOR DIFFERENT VALUES OF $q$ (NUMBER OF SOFT-DECISION BITS); GAUSS-MARKOV SOURCE WITH CORRELATION COEFFICIENT 0.9; NUMBERS IN Brackets Indicate the Optimal Performance Theoretically ATTAINABLE (OPTA) FOR THE GAUSS-MARKOV SOURCE $(\rho=0.9)$ AND DMC. (a) $r=2$ BITS/SAMPLE; DIMENSION $k=2$. (b) $r=2$ BITS/SAMPLE; DiMENSION $k=4$. SHCD RESULTS WERE OBTAINED FROM THE AUTHORS OF [18]; THEY ARE Also PlotTED IN [18, Fig. 8]

\begin{tabular}{c|c|c|c|c}
\hline Chan. & & & & \\
SNR & $q=1$ & $q=2$ & $q=3$ & $q=4$ \\
\hline$\infty$ & $13.52[19.25]$ & $13.52[19.25]$ & $13.52[19.25]$ & $13.52[19.25]$ \\
8 & $11.20[18.62]$ & $11.45[18.69]$ & $11.53[18.85]$ & $11.58[19.20]$ \\
6 & $8.92[17.35]$ & $9.72[17.63]$ & $9.94[18.00]$ & $9.99[18.93]$ \\
4 & $6.97[15.48]$ & $7.70[16.12]$ & $7.98[16.59]$ & $8.05[16.73]$ \\
3 & $6.03[14.46]$ & $6.86[15.28]$ & $7.14[15.73]$ & $7.21[15.85]$ \\
2 & $5.15[13.45]$ & $5.86[14.42]$ & $6.12[14.81]$ & $6.19[14.91]$ \\
1 & $4.34[12.51]$ & $5.06[13.54]$ & $5.25[13.87]$ & $5.31[13.96]$ \\
0 & $3.62[11.62]$ & $4.42[12.68]$ & $4.61[12.96]$ & $4.66[13.03]$ \\
-1 & $3.00[10.76]$ & $3.83[11.83]$ & $4.00[12.09]$ & $4.05[12.15]$ \\
-2 & $2.47[9.93]$ & $3.29[10.98]$ & $3.45[11.23]$ & $3.50[11.30]$ \\
-3 & $2.02[9.11]$ & $2.80[10.13]$ & $2.95[10.38]$ & $2.99[10.45]$ \\
\hline \multicolumn{4}{|c}{}
\end{tabular}

(a)

\begin{tabular}{c|c|c|c}
\hline Chan. & & & \\
SNR & $q=1$ & $q=2$ & SHCD [18] \\
\hline$\infty$ & $15.77[19.25]$ & $15.77[19.25]$ & 15.8 \\
8 & $13.35[18.62]$ & $13.75[18.69]$ & 12.44 \\
6 & $11.19[17.35]$ & $12.11[17.63]$ & 8.68 \\
4 & $8.89[15.48]$ & $10.02[16.12]$ & 5.63 \\
3 & $7.83[14.46]$ & $9.04[15.28]$ & 4.49 \\
2 & $6.91[13.45]$ & $8.07[14.42]$ & 3.58 \\
1 & $6.03[12.51]$ & $7.12[13.54]$ & 2.85 \\
0 & $5.20[11.62]$ & $6.25[12.68]$ & 2.28 \\
-1 & $4.45[10.76]$ & $5.42[11.83]$ & 1.82 \\
-2 & $3.77[9.93]$ & $4.67[10.98]$ & 1.45 \\
-3 & $3.17[9.11]$ & $3.97[10.13]$ & - \\
\hline
\end{tabular}

(b)

parameter 0.9, and with COVQ parameters $r=k=2$ and $r=2, k=4$, respectively. In this case, the source has high redundancy in the form of memory. In Table III(a), the results are obtained for $q$ ranging from 1 to 4; while in Table III(b), they are obtained for $q=1$ and 2 only. The largest improvement as $q$ varies from 1-4 in Table III(a) is 1.18 -dB SDR occurring at 3-dB channel SNR. In Table III(b), as $q$ increases from 1 to 2, the largest improvement is 1.21-dB SDR occurring at 3-dB channel SNR. The best coding gains at low channel SNRs are around 2 $\mathrm{dB}$ [Table III(a)] and $1.31 \mathrm{~dB}$ [Table III(b)] in channel SNR.

In Table III(b), we also compare the performance of the proposed soft-decision COVQ scheme with the soft Hadamard column decoder (SHCD) of [18]. The SHCD results in this table are for a fixed encoder whereas the results of the proposed scheme are for the case where both the encoder and decoder are optimized for the given channel SNR. Hence we find that even the hard-decision $(q=1)$ COVQ outperforms the SHCD of [18] with a fixed encoder (optimized for the clean channel). Thus the comparison in Table III(b) is not fair to [18]. In Fig. 2, we attempt to make a fair comparison. In this figure, we compare the proposed soft-decision COVQ scheme with the channel-optimized SHCD scheme (both encoder and decoder are optimized for the given channel SNR) for the Gauss-Markov source with $k=4, r=1$. In this case, we find that the proposed scheme with $q=4$ is comparable to

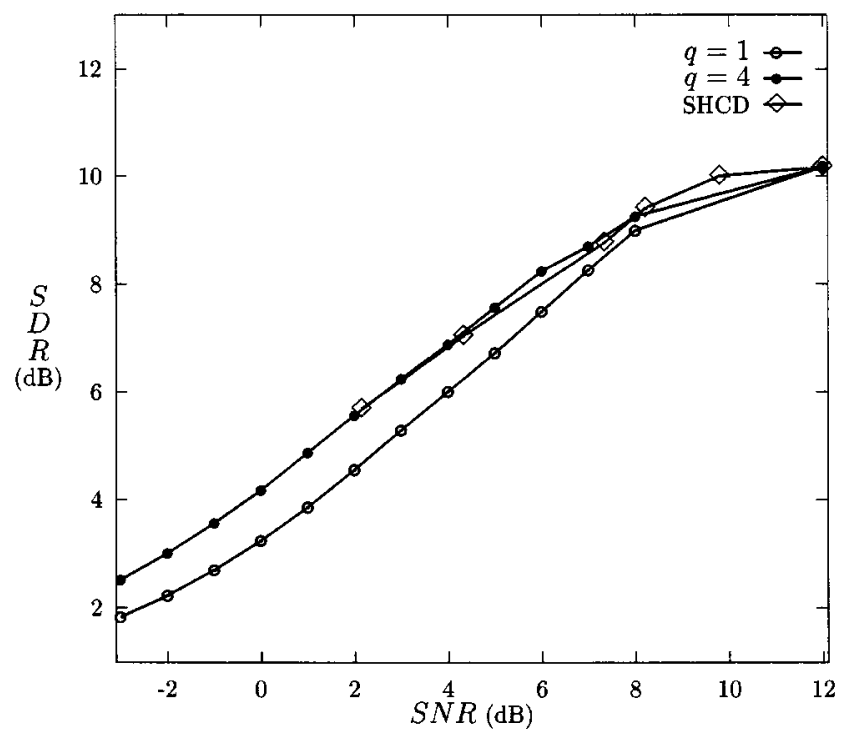

Fig. 2. Performances of COVQ system in AWGN channel with $q=1$ and $q=4$ compared with SHCD (cf. [18, Table 4]). Gauss-Markov source. $R=1$; $k=4$.

the channel-optimized SHCD. We observe that the decoder of the proposed scheme is just a simple table lookup while the decoder of the SHCD requires a weighted multiplication of $2^{k r} k$-dimensional vectors. Thus, the decoder computational complexity of the proposed scheme is substantially less than SHCD. However, the decoder memory storage of the proposed scheme is $2^{(q-1) k r}$ times more than the SHCD.

\section{ACGN Channels Without Noise Whitening}

We next investigate the COVQ system for a BPSK-modulated additive colored Gaussian noise (ACGN) channel. The system is the same as described in Section II [cf. (1) and Fig. 1] with the exception that the noise process $\left\{U_{n}\right\}$ is a $p$ th-order Gauss-Markov noise process described by

$$
U_{n}=\sum_{m=1}^{p} a_{m} U_{n-m}+\tilde{U}_{n}
$$

where $\left\{\tilde{U}_{n}\right\}$ is an i.i.d. (white) Gaussian process. Here, it is assumed that the all-pole filter described by (7) is stable, i.e., all the roots of the polynomial $A(z)=1-\sum_{m=1}^{p} a_{m} z^{-m}$ are within the unit circle.

Instead of using the traditional interleaving technique for such a channel, we propose to utilize the statistical characteristics of the correlated channel noise by incorporating them in the design of the COVQ. This results in a COVQ scheme exploiting both the channel memory as well as the channel soft-decision information. ${ }^{2}$ Like the AWGN case, the resulting discrete channel obtained by concatenating the modulator, ACGN channel, and the soft-decision demodulator, has $2^{k r}$ inputs and $2^{q k r}$ outputs. However, in this case, the channel transition matrix is nonsymmetric and the channel has memory from block to block; thus, its capacity cannot be easily obtained. To simplify this matter, we model this discrete channel as a block-memoryless channel

\footnotetext{
${ }^{2}$ In [14], a binary-input binary-output channel with memory was considered, thus only the channel memory was exploited by the COVQ design.
} 
TABLE IV

(a) CAPACITY $C$ (IN BITS/CHANNEL USE) OF $2^{k \cdot r}$-INPUT, $2^{q k r}$-OUTPUT DMC DERIVED FROM BPSK-MODULATED ACGN CHANNEL $\left(p=1, a_{1}=0.9\right)$ WITH $q$-BIT SOFT-DECISION DEMODULATION AND $k r=4 ; \Delta=\Delta$ (SNR) IS OPtimal Quantization STEP. (b) SOURCE SDR (IN DeCibels) Performance OF COVQ SYSTEM IN ACGN ChANNEL $\left(p=1, a_{1}=0.9\right)$ FOR DIFFERENT VALUES OF $q$ (NUMBER OF SOFT-DECISION BITS);

GausS-Markov Source With CORRELATION COEFFICIENT $0.9 ; r=2$ BITS/SAMPLE; DIMENSION $k=2$. NUMBERS IN BRACKETS INDICATE THE OPTIMAl PERFoRMANCE THEORETICALly ATTAINABLE (OPTA) FOR THE GAUSS-MARKOV SOURCE AND DMC

\begin{tabular}{c|c|c|c|c|c|c|c}
\hline \hline Channel & $q=1$ & \multicolumn{2}{|c|}{$q=2$} & \multicolumn{2}{c|}{$q=3$} & \multicolumn{2}{c}{$q=4$} \\
\cline { 2 - 8 } SNR & $C$ & $C$ & $\Delta$ & $C$ & $\Delta$ & $C$ & $\Delta$ \\
\hline \hline$\infty$ & 1.000 & 1.000 & 1.000 & 1.000 & 1.000 & 1.000 & 1.000 \\
8.0 & 0.956 & 0.993 & 0.585 & 0.999 & 0.466 & 1.000 & 0.198 \\
7.0 & 0.919 & 0.987 & 0.623 & 0.999 & 0.641 & 0.999 & 0.229 \\
6.0 & 0.870 & 0.978 & 0.660 & 0.997 & 0.629 & 0.998 & 0.266 \\
5.0 & 0.810 & 0.963 & 0.685 & 0.994 & 0.629 & 0.997 & 0.285 \\
4.0 & 0.744 & 0.941 & 0.729 & 0.989 & 0.616 & 0.994 & 0.298 \\
3.0 & 0.675 & 0.910 & 0.748 & 0.979 & 0.635 & 0.989 & 0.316 \\
2.0 & 0.608 & 0.870 & 0.816 & 0.963 & 0.641 & 0.981 & 0.329 \\
1.0 & 0.544 & 0.824 & 0.885 & 0.938 & 0.660 & 0.965 & 0.354 \\
0.0 & 0.482 & 0.773 & 1.048 & 0.903 & 0.654 & 0.941 & 0.373 \\
-1.0 & 0.425 & 0.720 & 1.154 & 0.857 & 0.691 & 0.905 & 0.398 \\
-2.0 & 0.372 & 0.662 & 1.248 & 0.803 & 0.729 & 0.859 & 0.429 \\
-3.0 & 0.324 & 0.602 & 1.373 & 0.743 & 0.798 & 0.804 & 0.460 \\
\hline
\end{tabular}
(a)

\begin{tabular}{c|c|c|c|c}
\hline SNR & $q=1$ & $q=2$ & $q=3$ & $q=4$ \\
\hline$\infty$ & $13.52[19.25]$ & $13.52[19.25]$ & $13.52[19.25]$ & $13.52[19.25]$ \\
8 & $11.51[18.72]$ & $12.31[19.17]$ & $13.03[19.24]$ & $13.18[19.25]$ \\
7 & $10.36[18.28]$ & $12.07[19.10]$ & $12.98[19.24]$ & $13.16[19.24]$ \\
6 & $9.19[17.69]$ & $11.27[18.99]$ & $12.53[19.22]$ & $12.95[19.23]$ \\
5 & $8.03[16.97]$ & $11.07[18.81]$ & $12.45[19.18]$ & $12.95[19.22]$ \\
4 & $6.95[16.17]$ & $10.23[18.54]$ & $11.89[19.12]$ & $12.56[19.18]$ \\
3 & $6.01[15.34]$ & $9.71[18.17]$ & $11.47[19.00]$ & $12.37[19.12]$ \\
2 & $5.17[14.53]$ & $9.06[17.69]$ & $11.05[18.81]$ & $11.91[19.02]$ \\
1 & $4.50[13.76]$ & $8.04[17.13]$ & $10.49[18.51]$ & $11.33[18.83]$ \\
0 & $3.92[13.02]$ & $7.78[16.52]$ & $9.89[18.09]$ & $10.61[18.54]$ \\
-1 & $3.40[12.33]$ & $7.15[15.88]$ & $9.26[17.53]$ & $10.26[18.11]$ \\
-2 & $3.05[11.66]$ & $6.57[15.18]$ & $8.54[16.88]$ & $9.73[17.56]$ \\
-3 & $2.68[11.02]$ & $6.00[14.46]$ & $7.84[16.16]$ & $9.04[16.89]$ \\
\hline
\end{tabular}

(b)

(a DMC with $2^{k r}$ inputs, $2^{q k r}$ outputs). Hence the noise memory from block-to-block is ignored while the memory within a block is considered. Therefore, as the blocklength $k r$ gets large, the model becomes more accurate. To determine the capacity, we estimate the $2^{k r} \times 2^{q k r}$ block transition matrix of the DMC using a long training sequence of colored noise. We then employ Blahut's algorithm [3] to calculate its capacity. As in Section II, the quantization step $\Delta$ is chosen to maximize capacity at each channel SNR. In Table IV(a), we present the channel capacity for the channel for different values of $q$ and for $k r=4$. Here, $p=1$ and $a_{1}=0.9$ (first-order Gauss-Markov noise) and the capacity is normalized by $k r$ to yield a unit of bits/channel use. The results indicate that soft-decision information provides very large gains in capacity. At low channel SNRs the capacity is increased by more than $100 \%$ from $q=1$ to $q=4$. Also, for the same SNR and the same $q$, the capacity of the quantized ACGN channel is always greater than the capacity of the quantized AWGN channel (cf. Table I).

The estimated channel transition matrix is then incorporated in the COVQ design algorithm [14]. Numerical results for the Gauss-Markov source over the ACGN channel are displayed in Table IV(b). It can been observed that for identical channel SNRs, substantial gains in SDR are achieved as $q$ increases from 1 to 4 . For low channel SNRs, the soft-decision gains are up to $6.8 \mathrm{~dB}$. Note that if a sufficiently long interleaver is used before and after channel transmission, the ACGN channel will be converted to an AWGN channel. Thus, comparing the results of Tables III(a) and IV(b), we remark that very large gains over channel interleaving are achieved-particularly for $q \geq 2$. For example, for $q=4$ and channel SNR $=-3 \mathrm{~dB}$, the SDR gain over the memoryless channel case is more than $6 \mathrm{~dB}$. This gain is due mainly to the higher capacity of the ACGN channel as compared to the AWGN channel [cf. Table I(a) and IV(a)].

\section{ACGN Channels With NoISE Whitening}

Another traditional method for dealing with an ACGN channel is to perform noise whitening [15]. Assume the noise parameters $\left\{a_{m}\right\}_{m=1}^{p}$ in (7) are known to the receiver. The channel output $Z_{n}$ is passed through a noise whitening filter $A(z)$, described by

$$
\hat{Z}_{n}=Z_{n}-\sum_{m=1}^{p} a_{m} Z_{n-m}
$$

where $\left\{Z_{n}\right\}$ are the filter input and $\left\{\hat{Z}_{n}\right\}$ are the filter output. Substituting (1) into (8), we get

$$
\begin{aligned}
\hat{Z}_{n} & =W_{n}+U_{n}-\sum_{m=1}^{p} a_{m}\left(W_{n-m}+U_{n-m}\right) \\
& =\left(W_{n}-\sum_{m=1}^{p} a_{m} W_{n-m}\right)+\left(U_{n}-\sum_{m=1}^{p} a_{m} U_{n-m}\right) \\
& =\left(W_{n}-\sum_{m=1}^{p} a_{m} W_{n-m}\right)+\tilde{U}_{n}
\end{aligned}
$$

where the last equality follows from (7).

Therefore, the noise-whitening filter $A(z)$ converts the ACGN $\left\{U_{n}\right\}$ in (7) into the AWGN $\left\{\tilde{U}_{n}\right\}$ in (9), while introducing the ISI: $-\sum_{m=1}^{p} a_{m} W_{n-m}$. Thus, the combination of the ACGN channel and the noise-whitening filter $A(z)$ is equivalent to an ISI channel with AWGN. Since the filter $A(z)$ is invertible, one may use the data processing theorem [6] to argue that the capacity of the ACGN channel is equal to the capacity of the induced ISI channel. When a soft-decision quantizer is introduced at the channel output, however, this no longer holds. Note that the noise-whitening filter $A(z)$ is introduced before the soft-decision quantizer.

\section{A. Scalar Soft-Decision Quantization System}

The questions we ask are the following. With a $q$-bit soft-decision quantizer at the channel output, does the introduction of the noise-whitening filter increase or decrease capacity? Furthermore, is the overall system performance improved or reduced with the noise-whitening filter?

In Table V(a), we present the capacity of the quantized ACGN channel with noise whitening $\left(\hat{Z}_{n}\right.$ is scalar quantized to $q$ bits). The COVQ performances are reported in Table V(b). As in Section III, we assume that the discrete channel is block 
TABLE $\mathrm{V}$

(a) CAPACITY $C$ (IN BITS/CHANNEL USE) OF $2^{k r}$-INPUT, $2^{q k r}$-OUTPUT DMC DERIVED FROM BPSK-MODULATED ACGN CHANNEL $\left(p=1, a_{1}=0.9\right)$ WITH NOISE WHITENING AND $q$-BIT SOFT-DECISION DEMODULATION AND $k r=4 ; \Delta=\Delta(\mathrm{SNR})$ IS OPTIMAL QUANTIZATION STEP. (b) SOURCE SDR (IN DECIBELS) PERFORMANCE OF COVQ SYSTEM WITH NOISE-WHITENING Filter; GAUSS-MARKOV SOURCE WITH CORRELATION COEFFICIENT 0.9; $r=2$ BITS/SAMPLE; DIMENSION $k=2$. NUMBERS IN BRACKETS INDICATE THE OPTIMAL PeRFormance THEORETICALly ATtAinable (OPTA) FOR THE GAUSS-MARKOV SOURCE AND DMC

\begin{tabular}{|c|c|c|c|c|c|c|c|}
\hline \multirow{2}{*}{$\begin{array}{l}\text { Channel } \\
\text { SNR }\end{array}$} & $q=1$ & \multicolumn{2}{|c|}{$q=2$} & \multicolumn{2}{|c|}{$q=3$} & \multicolumn{2}{|c|}{$q=4$} \\
\hline & $C$ & $\bar{C}$ & $\Delta$ & $C$ & $\Delta$ & $C$ & $\Delta$ \\
\hline & 1.000 & 1.000 & 0 & .000 & 0 & .000 & .00 \\
\hline 8.0 & 64 & 0.999 & 741 & 99 & & 00 & .104 \\
\hline 7.0 & 345 & 0.998 & 22 & 999 & 291 & 999 & 11 \\
\hline 6.0 & 624 & 0.997 & 10 & 10 & 316 & 98 & 13 \\
\hline 5.0 & 002 & 0.995 & 30 & & 0.335 & 97 & 0.15 \\
\hline 4.0 & 80 & 0.991 & & & 0 & & .16 \\
\hline 3.0 & 0.562 & 0.984 & 0.966 & 889 & 0.410 & 22 & 0.17 \\
\hline 2.0 & 16 & 0.971 & & 31 & 3 & 36 & .18 \\
\hline 1.0 & & 0.949 & & & 9 & & .15 \\
\hline 0.0 & 24 & 0.917 & & & $x$ & & .2 \\
\hline-1.0 & 617 & 0.873 & 0 & 11 & 0 & 26 & .22 \\
\hline-2.0 & 11 & 0.821 & 1.035 & 67 & 491 & 85 & 0.23 \\
\hline-3.0 & 0.505 & 0.763 & 1.066 & 0.814 & 0.516 & 0.834 & 0.2 \\
\hline & & & & & & & \\
\hline $\mathrm{NR}$ & $q=1$ & & $=2$ & & $\equiv 3$ & & \\
\hline$\infty$ & $52[19.2$ & 13. & $2[19.2$ & 13.5 & {$[19.25]$} & 13.52 & {$[19$.} \\
\hline 8 & {$[15$} & & {$[19$.} & 13. & [19.24] & 3.52 & {$[19.2$} \\
\hline 7 & $1[14.9$ & & {$[19$.} & 13. & {$[19.2$} & 13.52 & {$[19.2$} \\
\hline ( & $5[14.73$ & & $35[19.22]$ & 13. & {$[19.2$} & 13.51 & {$[19.2$} \\
\hline 5 & $7[14.46$ & & 30 [19.19] & 13. & {$[19.20]$} & 13.48 & {$[19.2$} \\
\hline 4 & $2[14$. & & 8 [19.14] & 13.2 & {$[19.1$} & 13.42 & {$[19$} \\
\hline 3 & $5[13$. & & {$[19.0$} & 13. & {$[19$.} & 13.26 & {$[19$} \\
\hline 8 & $9[13.7$ & & {$[18.90]$} & 12. & {$[19$} & 12.88 & {$[19$} \\
\hline 1 & $2[13.63$ & & {$[18.64]$} & & {$[18$.} & 12.21 & {$[18.9$} \\
\hline 0 & $8[13.52$ & & [18.25] & 11.1 & [18.59] & 11.54 & {$[18.7$} \\
\hline-1 & $5[13.44$ & 10. & $01[17.72]$ & 10.5 & [18.18] & 10.82 & {$[18.3$} \\
\hline-2 & $5[13.37$ & & 3 [17.10] & 9.71 & {$[17.65]$} & 10.38 & {$[17.8$} \\
\hline-3 & $7.86[13.29$ & 8.8 & $6[16.40]$ & 9.21 & {$[17.01$} & 9.71 & 17.2 \\
\hline
\end{tabular}

(b)

memoryless with blocklength $k r$. For system design, we reset the state, $\left(W_{-1}, W_{-2}, \cdots, W_{-p}\right)$, of the noise whitening filter at the beginning of each block to zeros. This leads to a block-based system which is simple to design and analyze. However, the "effective channel" (the combination of the ACGN channel and the noise-whitening filter) will not be exactly an ISI channel since the noise is not entirely whitened in the first $p$ samples of each block of $k r$ bits.

Comparing Tables IV(a) and V(a), we observe that the noise whitening filter does in fact increase capacity in all cases except for $q=1$ and high SNR. This is explained by the fact that a one-bit scalar quantizer is not effective for ISI channels. Consider as an example the case where $k r=2, p=1, a_{1}=0.9$, and $q=1$. Even though the transmitted signals, $\left(W_{0}, W_{1}\right)$, belong to

$$
\{(+1,+1),(-1,+1),(-1,-1),(+1,-1)\}
$$

the signal part of the received signals $\left(\hat{Z}_{0}, \hat{Z}_{1}\right)$ belong to

$$
\{(+1,+0.1),(-1,+1.9),(-1,-0.1),(+1,-1.9)\}
$$

due to the ISI in (9). Thus, it is apparent that in the case of ACGN channel with noise whitening, a vector soft-decision quantizer (VSDQ) will be more effective in capturing the channel memory than a scalar soft-decision quantizer. In the next sub-section, we propose two VSDQ methods.

\section{B. Vector Soft-Decision Quantization Systems}

We introduce two VSDQ systems: an unstructured VSDQ system using an LBG (Linde, Buzo, Gray) VQ [11] and a structured VSDQ. To derive the unstructured VSDQ, we obtain a sequence of $k r M$ noise samples derived from a Gauss-Markov process of order $p$. The noise process is obtained by passing a white Gaussian noise sequence through an all-pole filter described by (7). The noise samples are divided into blocks of size $k r$. We thus obtain $M$ noise vectors. Each of the noise vectors is added to each of the signal points on the constellation $\{-1,+1\}^{k r}$ of size $2^{k r}$. The $M 2^{k r}$ received vectors are passed through a noise-whitening filter described by the $k r \times k r$ matrix

$$
H=\left[\begin{array}{ccccccc}
1 & 0 & 0 & \cdots & \cdots & \cdots & 0 \\
-a_{1} & 1 & 0 & \cdots & \cdots & \cdots & 0 \\
-a_{2} & -a_{1} & 1 & \cdots & \cdots & \cdots & 0 \\
\vdots & \vdots & \vdots & \vdots & \vdots & \vdots & \vdots \\
0 & \cdots & 0 & -a_{p} & \cdots & -a_{1} & 1
\end{array}\right] .
$$

We thus obtain $M 2^{k r}$ VSDQ input vectors of dimension $k r$. These vectors are used as the training sequence in the design of an LBG-VQ [11] of size $2^{q k r}$ codevectors. Note that $q$ is the rate of the soft-decision vector quantizer. The LBG-VQ is designed assuming a squared-error distortion measure. This design criterion is chosen for convenience only and does not necessarily result in the best soft-decision vector quantizer in term of achieving the maximum capacity. Once the LBG-VQ is designed, a channel transition matrix of size $2^{q k r} \times 2^{k r}$ is determined by encoding each of the $M 2^{k r}$ received vectors. The capacity is then calculated using Blahut's algorithm.

The second VSDQ is designed based on the observation that at high SNR, the VSDQ input vectors are concentrated around the points $H \mathbf{W}$, where $\mathbf{W} \in\{-1,+1\}^{k r}$ is a $k r \times 1$ column vector. It is thus desirable to place codevectors near these points. To achieve this, we design a structured VSDQ in which each codevector is described by

$$
H \tilde{\mathbf{W}}
$$

where

$$
\begin{array}{r}
\tilde{\mathbf{W}} \in\left\{\frac{-\left(2^{q}-1\right) \Delta}{2}, \cdots, \frac{-3 \Delta}{2}, \frac{-\Delta}{2}, \frac{\Delta}{2}, \frac{3 \Delta}{2}, \cdots\right. \\
\left.\frac{\left(2^{q}-1\right) \Delta}{2}\right\}^{k r}
\end{array}
$$

Thus, the structured VSDQ codebook is a linear mapping (by $H$ ) of a uniform $k r$-dimensional grid (derived from $k r$ uses of a uniform scalar quantizer of step size $\Delta$ ). There are several advantages of the structured VSDQ as compared to the unstructured LBG-VQ. First, since the codebook depends only on one parameter, namely $\Delta$, obtaining the best codebook (for maximizing channel capacity) among those having the 
TABLE VI

CAPACITY $C$ (IN BITS/CHANNEL USE) OF $2^{k \cdot r}$-INPUT, $2^{q k r}$-OUTPUT DMC DERIVED FROM BPSK-MODULATED ACGN CHANNEL $\left(p=1, a_{1}=0.9\right.$ ) WITH NOISE WHITENING AND $q$-BIT VSDQ AND $k r=4$. (b) SOURCE SDR (IN DeCibels) PERFormance OF COVQ SYSTEM WITH STRUCTURED VSDQ; Gauss-Markov Source with CoRrelation CoefFicient $0.9 ; r=2$ BITS/SAMPLE; DIMENSION $k=2$. NUMBERS IN BRACKETS INDICATE THE OPtimal Performance THEORETICAlly ATtAINABLE (OPTA) FOR THE GAUSS-MARKOV SOURCE AND DMC

\begin{tabular}{c|c|c|c|c|c|c}
\hline \hline Channel & \multicolumn{2}{|c|}{ Unstructured VSDQ } & \multicolumn{3}{c}{ Structured VSDQ } \\
\cline { 2 - 7 } SNR & $q=1$ & $q=2$ & $q=3$ & $q=1$ & $q=2$ & $q=3$ \\
\hline \hline$\infty$ & 1.000 & 1.000 & 1.000 & 1.000 & 1.000 & 1.000 \\
8.0 & 0.999 & 0.999 & 0.999 & 0.998 & 0.999 & 1.000 \\
7.0 & 0.997 & 0.999 & 0.999 & 0.997 & 0.999 & 0.999 \\
6.0 & 0.994 & 0.998 & 0.998 & 0.994 & 0.997 & 0.998 \\
5.0 & 0.989 & 0.996 & 0.996 & 0.989 & 0.994 & 0.996 \\
4.0 & 0.980 & 0.992 & 0.994 & 0.980 & 0.989 & 0.994 \\
3.0 & 0.965 & 0.986 & 0.989 & 0.966 & 0.981 & 0.989 \\
2.0 & 0.941 & 0.972 & 0.979 & 0.944 & 0.967 & 0.980 \\
1.0 & 0.906 & 0.951 & 0.964 & 0.911 & 0.945 & 0.964 \\
0.0 & 0.860 & 0.919 & 0.940 & 0.869 & 0.915 & 0.940 \\
-1.0 & 0.802 & 0.875 & 0.906 & 0.816 & 0.876 & 0.905 \\
-2.0 & 0.736 & 0.823 & 0.861 & 0.756 & 0.828 & 0.860 \\
-3.0 & 0.666 & 0.764 & 0.808 & 0.690 & 0.772 & 0.806 \\
\hline
\end{tabular}

(a)

\begin{tabular}{c|c|c|c}
\hline SNR & $q=1$ & $q=2$ & $q=3$ \\
\hline$\infty$ & $13.52[19.25]$ & $13.52[19.25]$ & $13.52[19.25]$ \\
8 & $13.46[19.23]$ & $13.43[19.24]$ & $13.52[19.25]$ \\
7 & $13.45[19.22]$ & $13.42[19.24]$ & $13.52[19.24]$ \\
6 & $13.43[19.18]$ & $13.38[19.22]$ & $13.48[19.23]$ \\
5 & $13.36[19.12]$ & $13.29[19.18]$ & $13.37[19.20]$ \\
4 & $13.22[19.01]$ & $13.09[19.12]$ & $13.27[19.18]$ \\
3 & $12.92[18.84]$ & $12.68[19.02]$ & $13.02[19.12]$ \\
2 & $12.42[18.58]$ & $12.15[18.86]$ & $12.46[19.01]$ \\
1 & $11.68[18.18]$ & $11.81[18.59]$ & $11.94[18.82]$ \\
0 & $10.86[17.68]$ & $11.36[18.23]$ & $11.11[18.53]$ \\
-1 & $9.93[17.04]$ & $10.62[17.76]$ & $10.55[18.11]$ \\
-2 & $9.09[16.32]$ & $9.86[17.18]$ & $10.07[17.57]$ \\
-3 & $8.19[15.52]$ & $9.14[16.51]$ & $9.21[16.92]$ \\
\hline
\end{tabular}

(b)

structure $H \tilde{\mathbf{W}}$ is straightforward. Second, since the codebook is structured, the quantization process can be implemented straightforwardly using the Viterbi algorithm. ${ }^{3}$ The Viterbi trellis has $2^{q p}$ states with $2^{q}$ branches entering and leaving each state. To obtain the capacity of the discrete channel (which is derived from the BPSK modulator, ACGN channel, noise-whitening filter $H$, and the structured VSDQ), we use the training sequence approach described above to determine the channel transition matrix. From the channel transition matrix, the capacity is calculated from Blahut's algorithm. We note that with the training-based approach, the channel transition matrix of the discrete channel is often very sparse-especially for high SNR. Thus, the storage requirement for the channel transition matrix can be kept small even though $q k r$ is large. Furthermore, the complexity of calculating the Shannon capacity can be reduced when the matrix is sparse.

In Table VI(a), we present the capacity of the discrete channels derived from unstructured and structured VSDQ. Note that the structured VSDQ capacities are comparable to the unstructured VSDQ. Furthermore, for $q=1$, both unstructured and

${ }^{3}$ Note that in implementing the structured VSDQ encoder, we use the mean squared error criterion in the Viterbi algorithm. However, in the design of the structured VSDQ, we choose the value of $\Delta$ which maximizes channel capacity.
TABLE VII

(a) CAPACITY $C$ (IN BITS/CHANNEL USE) OF $2^{k \cdot r}$-INPUT, $2^{q k r}$-OUTPUT DMC DERIVED FROM BPSK-MODULATED ISI CHANNEL

$\left(p=2, a_{0}=0.407, a_{1}=0.815, a_{2}=0.417\right)$ WITH $q$-BIT STRUCTURED VSDQ AND $k r=4,6$, AND 8 . (b) SOURCE SDR (IN DECIBELS) PERFORMANCE OF COVQ SYSTEM WITH STRUCTURED VSDQ; GAUSS-MARKOV SOURCE WITH CORRELATION COEFFICIENT 0.9; $r=2$ BITS/SAMPLE; DimENSION $k=2,3$, AND 4

\begin{tabular}{c|c|c|c|c|c|c|c|c}
\hline \hline $\begin{array}{c}\text { Channel } \\
\text { SNR }\end{array}$ & \multicolumn{3}{|c|}{$k r=4$} & \multicolumn{3}{c|}{$k r=6$} & \multicolumn{2}{c}{$k r=8$} \\
\cline { 2 - 9 } & $q=1$ & $q=2$ & $q=3$ & $q=1$ & $q=2$ & $q=3$ & $q=1$ & $q=2$ \\
\hline \hline$\infty$ & 1.000 & 1.000 & 1.000 & 1.000 & 1.000 & 1.000 & 1.000 & 1.000 \\
10.0 & 0.826 & 0.859 & 0.882 & 0.866 & 0.895 & 0.935 & 0.890 & 0.918 \\
9.0 & 0.780 & 0.820 & $\mathbf{0 . 8 4 4}$ & 0.824 & 0.861 & 0.914 & 0.848 & 0.888 \\
8.0 & 0.731 & 0.775 & 0.803 & 0.777 & 0.821 & 0.888 & 0.800 & 0.851 \\
$\mathbf{7 . 0}$ & 0.681 & $\mathbf{0 . 7 2 9}$ & 0.758 & 0.725 & 0.776 & 0.858 & 0.746 & 0.809 \\
6.0 & 0.629 & $\mathbf{0 . 6 8 1}$ & 0.710 & 0.670 & 0.728 & 0.824 & 0.689 & 0.763 \\
5.0 & 0.577 & 0.632 & 0.661 & 0.614 & 0.676 & 0.787 & 0.631 & 0.715 \\
4.0 & 0.526 & 0.579 & 0.611 & 0.558 & 0.624 & 0.749 & 0.574 & 0.666 \\
3.0 & 0.478 & 0.528 & 0.560 & 0.506 & 0.571 & 0.711 & 0.522 & 0.619 \\
2.0 & 0.434 & 0.480 & 0.510 & 0.458 & 0.520 & 0.675 & 0.472 & 0.575 \\
1.0 & 0.393 & 0.434 & 0.463 & 0.415 & 0.471 & 0.640 & 0.427 & 0.533 \\
0.0 & 0.351 & 0.390 & 0.420 & 0.374 & 0.425 & 0.608 & 0.386 & 0.495 \\
-1.0 & 0.310 & 0.346 & 0.378 & 0.333 & 0.382 & 0.578 & 0.345 & 0.461 \\
-2.0 & 0.272 & 0.304 & 0.338 & 0.295 & 0.341 & 0.551 & 0.307 & 0.430 \\
-3.0 & 0.237 & 0.266 & 0.301 & 0.260 & 0.303 & 0.526 & 0.271 & 0.401 \\
\hline
\end{tabular}

\begin{tabular}{|c|c|c|c|c|c|c|c|c|c|}
\hline \multicolumn{10}{|c|}{ (a) } \\
\hline \multirow{2}{*}{$\begin{array}{l}\text { Chan. } \\
\text { SNR }\end{array}$} & \multicolumn{4}{|c|}{$k=2 ; r=2$} & \multicolumn{3}{|c|}{$k=3 ; r=2$} & \multicolumn{2}{|c|}{$k=4 ; r=2$} \\
\hline & $q=1$ & $q=2$ & $q=3$ & {$[19]$} & $q=1$ & $q=2$ & $q=3$ & $q=1$ & $q=2$ \\
\hline$\infty$ & 13.52 & 13.52 & 13.52 & - & 14.92 & 14.92 & 14.92 & 15.77 & 15.77 \\
\hline 10 & 10.21 & 10.71 & 10.86 & - & 12.50 & 12.35 & 12.85 & 13.48 & 13.49 \\
\hline 9 & 9.78 & 10.25 & 10.55 & 13.1 & 11.85 & 11.94 & 12.42 & 12.83 & 13.02 \\
\hline 8 & 9.59 & 9.75 & 9.98 & - & 11.11 & 11.57 & 11.90 & 12.16 & 12.45 \\
\hline 7 & 9.01 & 9.19 & 9.50 & 11.7 & 10.39 & 10.99 & 11.42 & 11.45 & 11.82 \\
\hline 6 & 8.40 & 8.91 & 9.19 & - & 9.80 & 10.32 & 10.96 & 10.83 & 11.29 \\
\hline 5 & 7.57 & 8.23 & 8.55 & 10.4 & 9.24 & 9.55 & 10.49 & 10.25 & 10.69 \\
\hline 4 & 7.23 & 7.41 & 7.75 & 一 & 8.75 & 9.13 & 9.97 & 9.62 & 10.18 \\
\hline 3 & 6.67 & 7.15 & 7.39 & 9.33 & 8.28 & 8.51 & 9.49 & 9.07 & 9.62 \\
\hline 2 & 6.07 & 6.59 & 6.84 & - & 7.77 & 7.81 & 8.86 & 8.54 & 9.16 \\
\hline 1 & 5.46 & 5.97 & 6.23 & 8.59 & 7.28 & 7.38 & 8.40 & 8.01 & 8.63 \\
\hline 0 & 4.84 & 5.31 & 5.58 & $\ldots$ & 6.72 & 6.79 & 7.94 & 7.44 & 8.20 \\
\hline-1 & 4.23 & 4.65 & 4.94 & - & 6.05 & 6.21 & 7.50 & 6.93 & 7.78 \\
\hline-2 & 4.06 & 4.33 & 4.56 & - & 5.43 & 5.59 & 7.02 & 6.35 & 7.25 \\
\hline-3 & 3.63 & 3.87 & 4.11 & - & 4.80 & 4.95 & 6.51 & 5.78 & 6.69 \\
\hline
\end{tabular}

structured VSDQ are better than the scalar quantizer (with and without noise whitening). For $q=3$, the structured VSDQ, the unstructured VSDQ, and the scalar soft-decision quantizer (with noise whitening) all yield similar results. The COVQ performance of the system assuming ACGN, noise whitening, and structured VSDQ is provided in Table VI(b). Considerable gains are observed over Tables IV(b) and V(b). Note that the structured VSDQ is chosen because of its moderate complexity and consistent performance.

\section{ISI CHANNELS}

We now consider an ISI channel modeled by

$$
Z_{n}=\left(\sum_{m=0}^{p} a_{m} W_{n-m}\right)+U_{n}
$$

where $W_{n} \in\{-1,+1\}$ is the channel input, $Z_{n}$ is the channel output, and $U_{n}$ is AWGN.

For purpose of simulation, we will consider the parameters $p=2$ and

$$
\left(a_{0}, a_{1}, a_{2}\right)=(0.407,0.815,0.407) .
$$


Such a filter has been considered in [15] and [19]. Note that the filter has a zero on the unit circle and hence linear equalization will result in very poor performance [15].

We now consider the structured VSDQ for the ISI channel. The capacity of the discrete channel is given in Table VII(a) for $k r=4,6$, and 8 . As before, the optimal values of $\Delta$ is chosen for each SNR. The COVQ performance is reported in Table VII(b). For comparison purposes, we have included the performance of the optimal soft-decision decoding method proposed by Skoglund [19] (referred to as the M1 scheme in [19]). Note that our scheme exploits the intrablock channel memory only; while Skoglund's technique exploits both the intrablock and interblock channel memories. Thus, Skoglund's scheme is superior for small blocklengths $(k r)$. However, our method has less computational complexity and can readily operate for higher blocklengths.

\section{CONCLUSIONS}

We presented and implemented a soft-decision COVQ system for BPSK-modulated AWGN, ACGN, and ISI Gaussian channels. The system, which consists of a COVQ scheme constructed for a discrete channel derived from the $q$-bit soft-decision demodulation of the Gaussian channels, exploits the channel soft information as well as the channel block memory in the case of the ACGN and ISI channels. The soft-decision demodulators are designed to maximize the capacity of the expanded channel which, in turn, results in improved COVQ performance. It is shown that the proposed scheme yields a considerably superior performance over COVQ schemes designed for hard-decision channels or channels that employ interleaving. Coding gains in SDR of up to $6.8 \mathrm{~dB}$ are achieved. Future work may address the study of soft-decision vector quantizers used in conjunction with error-control coding schemes

\section{REFERENCES}

[1] F. Alajaji and N. Phamdo, "Soft-decision COVQ for Rayleigh fading channels," IEEE Commun. Lett., vol. 2, pp. 162-164, June 1998.

[2] E. Ayanoglu and R. M. Gray, "The design of joint source and channel trellis waveform coders," IEEE Trans. Inform. Theory, vol. IT-33, pp. 855-865, Nov. 1987

[3] R. E. Blahut, Principles and Practice of Information Theory. Reading, MA: Addison-Wesley, 1988.

[4] N. Farvardin and V. Vaishampayan, "On the performance and complexity of channel-optimized vector quantizers," IEEE Trans. Inform. Theory, vol. 37, pp. 155-160, Jan. 1991

[5] S. Gadkari and K. Rose, "Transmission energy allocation with low peak-to-average ratio," IEEE Commun. Lett., vol. 1, pp. 166-168, Nov. 1997.

[6] R. G. Gallager, Information Theory and Reliable Communication. New York: Wiley, 1968

[7] A. Goldsmith and M. Effros, "Joint design of fixed-rate source codes and multiresolution channel codes," IEEE Trans. Commun., vol. 46, pp. 1301-1312, Oct. 1998

[8] K.-P. Ho and J. M. Kahn, "Joint design of a channel-optimized quantizer and multicarrier modulation," IEEE Trans. Commun., vol. 46, pp. 1254-1257, Oct. 1998

[9] V. Kafedziski and D. Morell, "Vector quantization over Gaussian channels with memory," in Proc. IEEE Int. Conf. Communications, Seattle, WA, 1995, pp. 1433-1437.

[10] H. Kumazawa, M. Kasahara, and T. Namekawa, "A construction of vector quantizers for noisy channels," Electron. Eng. Jpn., vol. 67-B, pp. 39-47, Jan. 1984
[11] Y. Linde, A. Buzo, and R. M. Gray, "An algorithm for vector quantization design,” IEEE Trans. Commun., vol. COM-28, pp. 84-95, Dec. 1980.

[12] F.-H. Liu, P. Ho, and V. Cuperman, "Sequential reconstruction of vector quantized signals transmitted over Rayleigh fading channels," in Proc. IEEE Int. Conf. Communications, New Orleans, LA, 1994, pp. 23-27.

[13] D. J. Miller and M. Park, "A sequence-based approximate MMSE decoder for source coding over noisy channels using discrete hidden Markov models," IEEE Trans. Commun., vol. 46, pp. 222-231, Feb. 1998.

[14] N. Phamdo, F. Alajaji, and N. Farvardin, "Quantization of memoryless and Gauss-Markov sources over binary Markov channels," IEEE Trans. Commun., vol. 45, pp. 668-675, June 1997.

[15] J. G. Proakis, Digital Communications, 2nd ed. New York: McGrawHill, 1989.

[16] P. L. Secker and P. O. Ogunbona, "Methods of channel-optimized trellis source coding for the AWGN channel," in Proc. IEEE Int. Symp. Information Theory, Trondheim, Norway, June 1994, p. 236.

[17] C. E. Shannon, "A mathematical theory of communication," Bell Syst. Tech. J., vol. 27, pp. 379-423, pp. 623-656, Oct. 1948

[18] M. Skoglund and P. Hedelin, "Hadamard-based soft-decoding for vector quantization over noisy channels," IEEE Trans. Inform. Theory, vol. 45, pp. 515-532, Mar. 1999

[19] M. Skoglund, "Soft decoding for vector quantization over noisy channels with memory," IEEE Trans. Inform. Theory, vol. 45, pp. 1293-1307, May 1999.

[20] G. Taricco, "On the capacity of the binary input Gaussian and Rayleigh fading channels," Eur. Trans. Telecommun., vol. 7, no. 2, Mar./Apr. 1996.

[21] V. Vaishampayan and N. Farvardin, "Joint design of block source codes and modulation signal sets," IEEE Trans. Inform. Theory, vol. 36, pp. 1230-1248, July 1992.

[22] M. O. Skoglund, "Bit-estimate based decoding for vector quantization over noisy channels with intersymbol interference," IEEE Trans Commun., vol. 48, pp. 1309-1317, Aug. 2000.

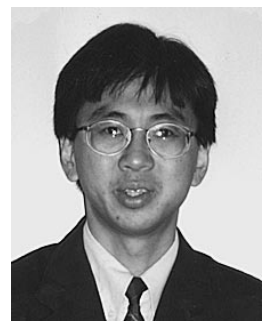

Nam Phamdo (S'88-M'93-SM'98) was born in Saigon, Vietnam, on February 20, 1966. He received the B.S., M.S., and Ph.D. degrees in electrical engineering from the University of Maryland at College Park in 1988, 1989, and 1993, repectively.

In 1993, he joined the Department of Electrical Engineering at the State University of New York (SUNY) at Stony Brook as an Assistant Professor. He became an Associate Professor in 1999. He has held visiting positions at Nippon Telegraph and Telephone (NTT) Human Interface Laboratories, Tokyo, Japan, and at the U.S. Army Research Laboratory at Aberdeen Proving Ground, MD. His research interest include speech coding and enhancement, joint source-channel coding, trellis coding, and turbo coding.

Prof. Phamdo is a member of Eta Kappa Nu.

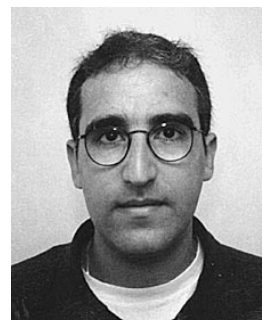

Fady Alajaji (S'90-M'94-SM'00) was born in Beirut, Lebanon, on May 1, 1966. He received the B.E. degree (with Distinction) from the American University of Beirut, Lebanon, and the M.S. and $\mathrm{Ph} . \mathrm{D}$. degrees from the University of Maryland at College Park, all in electrical engineering, in 1998, 1990, and 1994, respectively. He held a Postdoctoral appointment in 1994 at the Institute for Systems Research, University of Maryland.

In 1995, he joined the Department of Mathematics and Statistics at Queen's University, Kingston, ON, Canada, where he is currently an Assistant Professor of Mathematics and Engineering. Since 1997, he was cross-appointed as an Assistant Professor in the Department of Electrical and Computer Engineering at Queen's University. He served as the Co-Chair of the 1999 Canadian Workshop on Information Theory held in Kingston, ON, Canada. His research interests include information theory, digital communications, error control coding, joint source-channel coding, and data compression. 